\title{
Embolization of medium-sized vessels with the penumbra occlusion device: evaluation of anchoring function
}

\author{
Kenichi Kato ${ }^{*}$, Kazuya Kawashima, Tomohiro Suzuki, Makoto Hamano, Sohei Yoshida and Kunihiro Yoshioka
}

\begin{abstract}
Background: The penumbra occlusion device (POD) is a recently developed metallic coil with a unique anchor segment. The purpose of this study was to investigate the anchoring function of the POD for embolization of medium-sized vessels in detail.
\end{abstract}

Materials and methods: We reviewed a series of cases of proximal embolization of medium-sized vessels in which the POD was used. Endovascular outcomes and complications were assessed. The distance between the distal end of the first-indwelled POD and the microcatheter tip was defined as the "landing distance," and this was also evaluated via fluoroscopic analysis.

Results: POD deployment was successful in 17 of 18 patients. The median landing distance was $9.6 \mathrm{~mm}$, and no distal POD migration was observed after the formation of anchor loops.

Conclusions: The specific anchoring function of the POD enables effective proximal occlusion of medium-sized vessels.

Keywords: Embolization, Penumbra occlusion device, Interventional radiology

\section{Background}

Proximal occlusion using metallic coils is sometimes technically challenging. A bigger coil in diameter relative to target vessel is difficult to form the anchor. On the other hand, a smaller coil relative to target vessel is possible to tightly compact, but it has high risk of migration. The penumbra occlusion device (POD; Penumbra Inc., Alameda, California, USA) is a unique hybrid coil, which has specific anchor function to achieve occlusion of medium-sized vessels. Specifically, the distal tip of the POD device is designed stiffer and larger in diameter than the rest of the device. Thus, the distal end of a POD can serve as an anchor, and the rest of the device can be securely stuffed into the anchor segment
(Jambon et al. 2017; Petitpierre et al. 2016; Spiotta et al. 2016). Our aim was to retrospectively assess the safety and efficacy of embolization and to especially investigate the anchoring function of the POD for embolization of medium-sized vessels.

\section{Materials and methods \\ Patients}

The Institutional Review Board approved this retrospective study. We obtained patient information from our hospital database. Between September 2016 and November 2018, embolization using the POD was performed in 61 patients. Among these patients, cases using the POD for mere coil packing without anchoring function were excluded. A total of 18 patients (median age: 69 years;

\footnotetext{
* Correspondence: kkato@iwate-med.ac.jp

Department of Radiology, Iwate Medical University, 1-1-1 Idaidori, Shiwa-gun, Yahaba-cho, Iwate Prefecture 028-3694, Japan
}

\section{Springer Open}

(c) The Author(s). 2020 Open Access This article is licensed under a Creative Commons Attribution 4.0 International License, which permits use, sharing, adaptation, distribution and reproduction in any medium or format, as long as you give appropriate credit to the original author(s) and the source, provide a link to the Creative Commons licence, and indicate if changes were made. The images or other third party material in this article are included in the article's Creative Commons licence, unless indicated otherwise in a credit line to the material. If material is not included in the article's Creative Commons licence and your intended use is not permitted by statutory regulation or exceeds the permitted use, you will need to obtain permission directly from the copyright holder. To view a copy of this licence, visit http://creativecommons.org/licenses/by/4.0/. 
age range: 42-80; 11 men, 7 women) were selected for inclusion in the study. The characteristics of the patients are listed in Table 1.

\section{Interventional procedure}

Under local anesthesia, a femoral puncture using the Seldinger technique and selective catheterization were performed in all patients. In the case of a pseudoaneurysm (PAN), parent arterial embolization was planned using the "isolation technique"; namely, the embolization of vessels both distal and proximal to the PAN was performed. The diameter of the target vessel was calculated from selective angiography. The size of a POD used was chosen based on the size of the target vessel. For diameter of a target vessel of $3.25-4 \mathrm{~mm}, 4 \mathrm{~mm}-5 \mathrm{~mm}, 5 \mathrm{~mm}-6 \mathrm{~mm}$ and $6 \mathrm{~mm}$ $8 \mathrm{~mm}$ : POD 4, POD 5, POD 6, and POD 8 was chosen, respectively. The POD was delivered through a 0.025 -in. microcatheter (Progreat $\Omega$, Terumo, Tokyo, Japan). Additional PODs or other metallic coils were used until hemostasis was obtained on a case-by-case basis.

\section{Measured outcomes}

Technical outcomes and complications were assessed. The fluoroscopic movie, if available, was also reviewed to assess the anchoring function

Table 1 Patient characteristics and medical history

\begin{tabular}{lll}
\hline Case & Disease & Indication of embolization \\
\hline 1 & IPMN & PAN after PpPD \\
2 & Insulinoma & PAN after PpPD \\
3 & PAVM & Occlusion of AV shunt \\
4 & PAVM & Occlusion of AV shunt \\
5 & Renal aneurysm & Renal aneurysmal rupture \\
6 & Bile duct cancer & PAN after PpPD \\
7 & Bile duct cancer & PAN after PpPD \\
8 & Cholecystitis & PAN after cholecystectomy \\
9 & IPMN & PAN after PpPD \\
10 & Bile duct cancer & PAN after PpPD \\
11 & Renal aneurysm & Renal aneurysmal rupture \\
12 & Papilla vater cancer & PAN after PpPD \\
13 & Bile duct cancer & PAN after PpPD \\
14 & Bile duct cancer & PAN after HPD \\
15 & PAVM & Occlusion of AV shunt \\
16 & Pancreatitis & Splenic PAN \\
17 & Bile duct cancer & PAN after PpPD \\
18 & Hepatic aneurysm & Hepatic aneurysm \\
\hline
\end{tabular}

IPMN intraductal papillary mucinous neoplasm, PAN pseudoaneurysm, PAVM pulmonary arteriovenous malformation, $P p P D$ pylorus preserving pancreatoduodenectomy, AV shunt arteriovenous shunt during the deployment of the first indwelling POD. A POD comprised an anchoring segment and a packing segment. During POD deployment, the anchoring segment began to stabilize the artery following the packing of the remnant coil. Thus, the distance between the distal end of the anchor segment and the microcatheter tip was defined as the "landing distance," and this was evaluated via fluoroscopic analysis (Figs. 1 and 2).

\section{Results}

Proximal arterial embolization using the anchoring function of the POD was performed in 18 patients. The target vessels in the 18 patients were as follows: proper hepatic artery $(n=9)$, right hepatic artery (RHA, $n=3)$, pulmonary artery $(n=3)$, renal artery $(n=2)$, and splenic artery $(n=1)$. POD deployment was successful in 17 of 18 patients. In a patient with a huge aneurysm of the RHA, the isolation technique was planned, and embolization of the distal RHA using the POD was successful. However, the POD could not be deployed in the proximal RHA because the proximal landing distance of the RHA was short enough to preserve left hepatic flow, and the POD could not be deployed in this patient. Instead of a POD, a vascular plug was placed in the proximal RHA (Fig. 3). Fluoroscopic analysis during deployment of the first indwelling POD was assessed in 15 patients. The target-vessel diameter in each patient was between 1.7 and $7.0 \mathrm{~mm}$, and the optimal POD size was chosen in each case. The median landing distance was $9.6 \mathrm{~mm}$ (range 1.4-20.9) (Table 2). After the distal end of the POD formed anchor loops that adhered to the vessel wall, no distal migration was observed on fluoroscopic analysis.

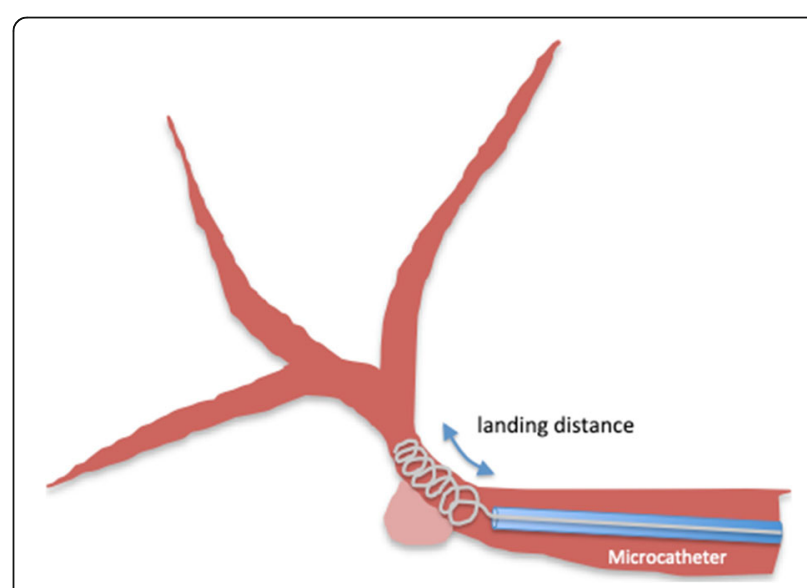

Fig. 1 The distance between the distal end of the first penumbra occlusion device and the microcatheter tip was defined as the "landing distance," and this was evaluated via fluoroscopic analysis 

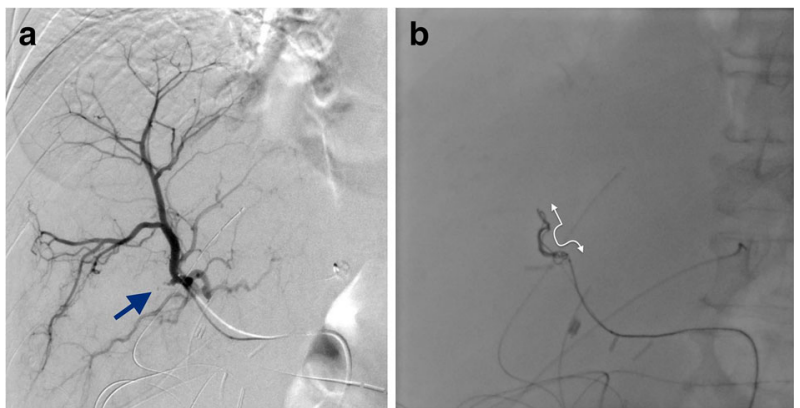

Fig. 2 a Three weeks after pylorus preserving pancreatoduodenectomy, bleeding from the cholecystic arterial stump (arrow) occurred. b Embolization around the cholecystic arterial stump using a 4-mm penumbra occlusion device. The two-way arrow shows the landing distance
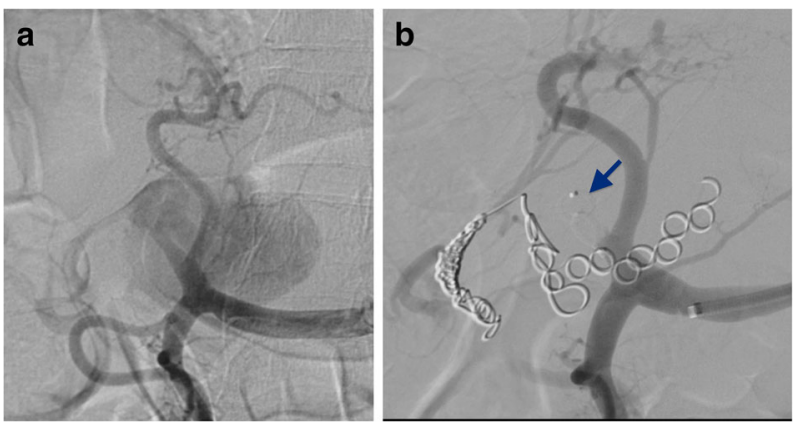

Fig. 3 a A huge aneurysm of the right hepatic artery. b A vascular plug (arrow), instead of a penumbra occlusion device, was placed in the proximal right hepatic artery

Table 2 Endovascular outcomes

\begin{tabular}{llllll}
\hline Case & Embolized artery & Diameter of target artery $(\mathrm{mm})$ & First indwelling POD & Landing distance (mm) & Additional coils \\
\hline 1 & Replaced RHA & 4.7 & $6 \mathrm{~mm} \times 50 \mathrm{~cm}$ & 16.6 & Ruby coil \\
2 & RHA & 3.3 & $4 \mathrm{~mm} \times 30 \mathrm{~cm}$ & 10.0 & Ruby coil \\
3 & PA & 3.8 & $4 \mathrm{~mm} \times 30 \mathrm{~cm}$ & 6.3 & Ruby coil \\
4 & PA & 2.9 & $4 \mathrm{~mm} \times 30 \mathrm{~cm}$ & 11.8 & - \\
5 & RA & 4.3 & $4 \mathrm{~mm} \times 30 \mathrm{~cm}$ & 20.9 & Ruby coil \\
6 & CHA & 3.5 & $4 \mathrm{~mm} \times 30 \mathrm{~cm}$ & 6.3 & Ruby coil \\
7 & CHA & 7.0 & $8 \mathrm{~mm} \times 60 \mathrm{~cm}$ & 16.7 & Ruby coil \\
8 & RHA & 4.1 & $4 \mathrm{~mm} \times 30 \mathrm{~cm}$ & 10.0 & POD, Ruby coil \\
9 & RHA & 3.0 & $4 \mathrm{~mm} \times 30 \mathrm{~cm}$ & $a$ & Ruby coil \\
10 & CHA & $5 \mathrm{~mm} \times 30 \mathrm{~cm}$ & $a$ & POD, Ruby coil \\
11 & RA & 5.7 & $4 \mathrm{~mm} \times 30 \mathrm{~cm}$ & 20.2 & POD, Ruby coil \\
12 & CHA & 2.0 & $4 \mathrm{~mm} \times 30 \mathrm{~cm}$ & 6.9 & POD, Ruby coil \\
13 & CHA & 1.7 & $4 \mathrm{~mm} \times 30 \mathrm{~cm}$ & 9.6 & Ruby coil \\
14 & CHA & 3.3 & $4 \mathrm{~mm} \times 30 \mathrm{~cm}$ & 8.1 & POD, Ruby coil \\
15 & PA & 2.5 & $5 \mathrm{~mm} \times 30 \mathrm{~cm}$ & 1.4 & POD \\
16 & SA & 5.2 & $4 \mathrm{~mm} \times 30 \mathrm{~cm}$ & 8.6 & POD, Ruby coil \\
17 & CHA & 4.7 & $5 \mathrm{~mm} \times 30 \mathrm{~cm}$ & 8.6 & POD, Ruby coil \\
18 & RHA & 4.2 & $5 \mathrm{~mm} \times 30 \mathrm{~cm}$ & bOD, AVP \\
\hline
\end{tabular}

RHA right hepatic artery, CHA common hepatic artery, RA renal artery, PA pulmonary artery, AVP Amplatzer vascular plug, SA splenic artery afluoroscopic movie was not available, and anchoring distance could not be verified

${ }^{b}$ POD could not be deployed due to unsuccessful anchoring 


\section{Discussion}

For embolization in cases, such as PAN and arteriovenous shunt, the proximal occlusion of target vessels is challenging. Special attention must be paid during the procedure to avoid the migration of the embolic device. The introduction of a POD is expected to solve this problem. The structure of the POD is unique with the distal end designed to work as an anchoring segment that enables proximal occlusion. Based on our fluoroscopic analysis, the median landing distance was $9.6 \mathrm{~mm}$, and no POD migration was observed after the anchoring segment was successfully deployed. However, the deployment of the anchoring segment was unsuccessful in one case in our study. Presumably, an insufficient landing distance relative to the arterial flow caused the unsuccessful POD deployment. Thus, in this case, a vascular plug might be an alternative embolization technique. Stent grafting is also another technique for shielding a PAN (Sapna et al. 2011). Nevertheless, when the anchoring segment of the POD is successfully deployed, tight coil packing is possible. Moreover, the POD has specific advantages of navigation in tortuous vessels using a microcatheter (Jambon et al. 2018). Therefore, the POD seems to be an effective tool for the proximal occlusion of medium-sized vessels in various targets.

\section{Conclusions}

Our fluoroscopic analysis indicates that the specific anchoring function of the POD enables effective proximal occlusion for medium-sized vessels in most cases. However, anchor formation is occasionally difficult in some situations, and other techniques are necessary for safer complete occlusion.

\section{Abbreviations \\ POD: penumbra occlusion device; PAN: pseudoaneurysm; RHA: right hepatic artery}

\section{Acknowledgements}

Not applicable.

\section{Authors' contributions}

Study Conception and Design: KK, KY. Data Collection and Analysis: KK, KK, TS, and MH. Interpretation: KK, MH. Manuscript Writing: KK. All authors read and approved the final manuscript.

\section{Funding}

No funding was obtained for this research.

\section{Availability of data and materials}

Please contact author for data requests.

\section{Ethics approval and consent to participate}

It was a retrospective study with research ethics board approval. This study was approved by the Iwate Medical University and a waiver of consent was obtained (MH2019-059).

\section{Consent for publication}

This was a retrospective study; all patients underwent clinically indicated transarterial embolization. Informed consent was waived by the local ethics committee. All figures used in preparing the manuscript contain no

individual or identifiable personal data.

\section{Competing interests}

The authors declare that they have no competing interests.

Received: 23 November 2019 Accepted: 17 March 2020

Published online: 04 May 2020

\section{References}

Jambon E, Hocquelet A, Petitpierre F et al (2018) Proximal embolization of splenic artery in acute trauma: comparison between penumbra occlusion device versus coils or Amplatzer vascular plug. Diagn Interv Imaging 99:801808

Jambon E, Petitpierre F, Brizzi V et al (2017) Proximal occlusion of medium-sized vessels with the penumbra occlusion device: a study of safety and efficacy. Cardiovasc Intervent Radiol 40:210-215

Petitpierre F, Lasserre AS, Tricaud E et al (2016) Proximal embolization of the splenic artery with a penumbra occlusion device (POD): a novel occlusion technique for blunt splenic injuries. Cardiovasc Intervent Radiol 39:309-312

Sapna P, Patel J, McPherson S et al (2011) Hemorrhagic complications after Whipple surgery: imaging and radiologic intervention. AJR 196:192-197 Spiotta AM, Turner RD, Chaudry Ml et al (2016) Carotid sacrifice with a single penumbra occlusion device: a feasibility study in a swine model. J Neurointerv Surg 8:99-102

\section{Publisher's Note}

Springer Nature remains neutral with regard to jurisdictional claims in published maps and institutional affiliations.

\section{Submit your manuscript to a SpringerOpen ${ }^{\circ}$ journal and benefit from:}

- Convenient online submission

- Rigorous peer review

- Open access: articles freely available online

High visibility within the field

- Retaining the copyright to your article

Submit your next manuscript at $\boldsymbol{\nabla}$ springeropen.com 